\title{
The Local Distribution of the Strain in Plastically Deformed Aluminium Crystals (4, 5 th Report). Analyses of Slip Bands and Clustered Slips by Means of X-Ray Diffraction Microscopy*
}

\author{
By Zenji Nishiyama** and Satohiro Hayami***
}

\begin{abstract}
Slip bands and clustered slips in deformed aluminium single crystals have been analysed by the Berg-Barrett method. From the results it has been concluded that (i) local latice curvature is present at the slip bands and also the twists whose axes are perpendicular to the slip planes, and that (ii) there are snall regions of secondary slip in the primary slip region, and vice versa, that (iii) the strain due to deformation is concentrated near the boundaries of the slip regions, and that (iv) there are striations denoted by $S_{1}$ which are straight and inclined by several degrees to the primary slip trace.
\end{abstract}

(Received April 20, 1960)

\section{Introduction}

In the previous papers, ${ }^{(1)(2)(3)}$ the present writers reported some of the results obtained by X-ray diffraction microscopy (Berg-Barrett method) using deformed aluminium crystals. While the optical micrograph shows only the surface uneveness of the deformed crystal, the X-ray diffraction micrograph shows directly the local distribution of the strain such as a misorientation, and lattice distortion of the crystal. The typical strain patterns taken from the uniformly slipped region could be classified generally into two categories, striations $\mathrm{K}$ and $\mathrm{S}$, which were related and nearly parallel to deformation bands and slip bands, respectively.

After the detailed analysis on striations $\mathrm{K}$ had been carried out in their last work, ${ }^{(3)}$ a study was further made in detail about striations $\mathrm{S}$ and also about the clustered slip region showing four kinds of striations $\left(\mathrm{S}_{1}, \mathrm{~S}_{2}, \mathrm{~S}^{\prime}, \mathrm{S}_{\mathrm{b}}\right)^{(2)}$ related to the active slips ${ }^{(2)}$. The detailed analyses of these striations will be given in the present paper.

\section{Experimental Procedure and Specimens}

The $\mathrm{X}$-ray apparatus is almost the same as previously used(1)(2). Since the angular range in which the striation pattern appears is far narrower for $S$ than for $\mathrm{K}$, a finely adjustable screw for the glancing angle (accuracy $2^{\prime}$ ) was fitted to the newly designed Berg-Barrett camera. In this camera, the specimen and

** Institute of Scientific and Industrial Reşarch, Osaka University.

*** Division for Research of Engineering, Graduate School, Osaka University. Now ai Tokyo Research Institute of the Yawata Iron and Steel Co., Ltd.

* A part of this paper was pressnted at the 1958 Autumn meeting of The Japan Institute of Metals and the other part the 1959 Spring meeting of The Physical Society of Japan, and was published in originally in Japanese in the Journal of the Japan Institute of Metals, 23(1959), 53; 24 (1960), 260.

(1) Z. Nishiyama and M. Yamamoto: Memoirs of the Inst. Sci. Ind. Res., Osaka Univ., 11 (1954), 163.

(2) M. Yamamoto, S. Hayami and Z. Nishiyama: Ibid, 13 (1956), 11.

(3) Z. Nishiyama and S. Hayami: Ibid, 15 (1958), 129. the plate cassette can be rotated together about an axis parallel to the incident beam so that the direction of the rotating axis of the specimen may be set either parallel or perpendicular to the longitudinal direction of the X-ray line source which is vertical in the present experiment.

In the "parallel" case the sensitivity in the misorientation of the crystal is $20^{\prime}$ in the top-to-bottom direction and $1^{\prime} \sim 2^{\prime}$ in the right-to-left direction in the photographs shown, while in the "perpendicular" case the sensitivity is vice versa. The photographic plates used are fine-grained (Kodak spectroscopic plate, 548-0).

The specimens are single crystals made by the stressannealing method from $99.98 \%$ or $99.99 \%$ purity aluminium plates denoted by $A_{0}$ and $A_{4}$, respectively. The size of the specimen is given in Table 1.

The specimen was electropolished without any previous mechanical polishing and then stretched in the length direction for plastic deformation. Before the deformation, the orientation of the crystal had been determined by the usual Laue method with graphical estimation. In Table 1 are given the determined orientation data of some of the specimens used.

\section{Results of $\mathbb{E x p e r i m e n t s ~ a n d ~ D i s c u s s i o n s ~}$}

All X-ray micrographs in the present paper are reproduced in the same way as stated previously, $(1)(2)(3)$ the X-ray beam being incident from left to right, so that each micrograph corresponds to the aspect of the irradiated surface of the specimen.

In these photographs, an arrow mark $(\leftrightarrow)$ indicates the direction of elongation, $\alpha$ is the glancing angle at the specimen surface, and dark areas correspond to the specimen portions which reflected X-rays strongly. The strong reflection, of course, implies that the crystal portion concerned is oriented with the angle favourable for Bragg reflection or that the extinction effect at that portion is reduced due to lattice distortion.

\section{(a) Slip bands}

Fig. 1 (a) is an optical micrograph taken of 
Table 1

\begin{tabular}{|c|c|c|c|c|c|c|c|c|c|c|c|}
\hline \multirow{2}{*}{$\begin{array}{c}\text { Specimen } \\
\text { No. }\end{array}$} & \multirow{2}{*}{$\begin{array}{l}\text { Size } \\
\left(\mathrm{mm}^{3}\right)\end{array}$} & \multicolumn{3}{|c|}{ Tension Axis } & \multicolumn{3}{|c|}{ Normal of Top Surface } & \multicolumn{4}{|c|}{ Plane \& Direction of Primary Slip } \\
\hline & & $\alpha$ & $\beta$ & $r$ & $\alpha$ & $\beta$ & $r$ & $\theta$ & $\lambda$ & $\eta$ & s \\
\hline $\mathrm{A}_{4-148 \mathrm{~B}}$ & $1.0 \times 10 \times 80$ & $84^{\circ}$ & $19^{\circ}$ & $72^{\circ}$ & $72^{\circ}$ & $75^{\circ}$ & $24^{\circ}$ & $48^{\circ}$ & $42^{\circ}$ & $6^{\circ}$ & $66^{\circ}$ \\
\hline$A_{0}-\mathrm{IV}-4$ & $0.5 \times 8 \times 70$ & $72.4^{\circ}$ & $19.5^{\circ}$ & $82.1^{\circ}$ & $52.0^{\circ}$ & $72.5^{\circ}$ & $43.4^{\circ}$ & $50.2^{\circ}$ & $40.2^{\circ}$ & $6^{\circ}$ & $72.5^{\circ}$ \\
\hline
\end{tabular}

$\theta:$ Angle between the tension axis and the normal of the slip plane,

$\eta$ : Angle between the iension axis and the slip direction,

$\lambda$ : Angle batween the slip direction and the projection of the tension axis on the slip plane,

$s$ : Angle between the slip direciion and the normal of the specimen surface.

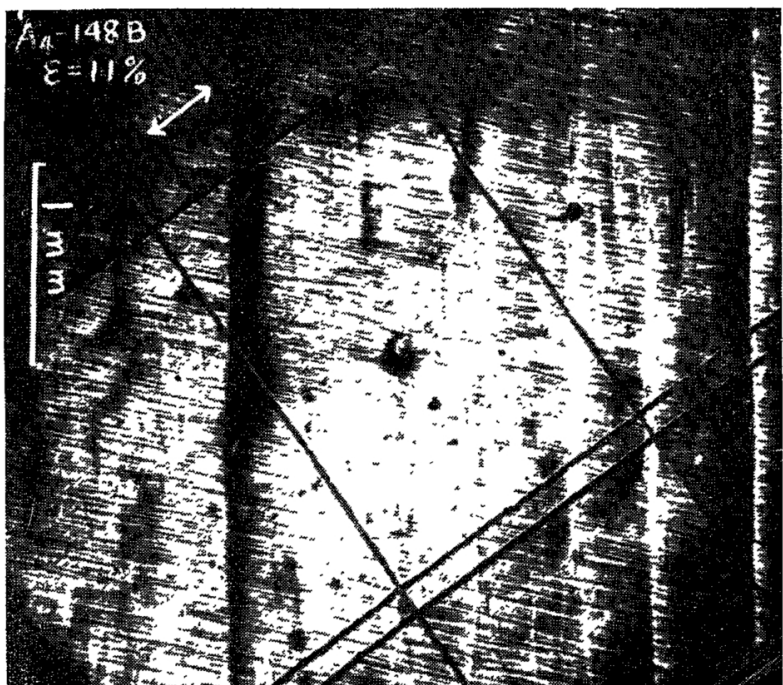

(a)
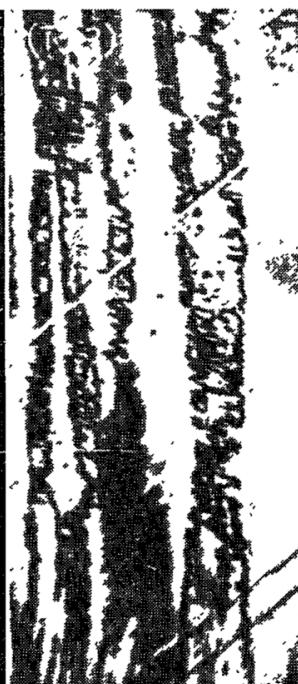

(b) (11) 3 ) reflection, $\alpha=\overline{4} 95^{\circ}$

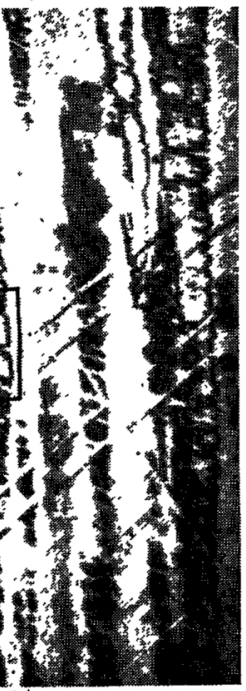

$\times 21$

Fig. 1 Optical (a) and X-ray (b) micrographs at the same portion in the specimen 148 B (elongation $\varepsilon=11 \%$ ). Striations $\mathrm{K}$ and $\mathrm{S}$ are parallel to the deformation and slip bands, respectively.

Specimen 148B elongated by $11 \%$ and (b) is an

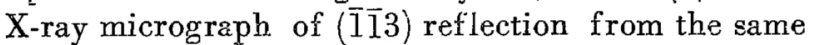
portion as (a). Oblique straight lines seen in these photographs are scratch marks made for reference after the elongation. In this specimen, as seen in (a), the slip bands are uniform covering the whole area, and the deformation bands show comparatively coarse distribution, while the X-ray micrograph shows both striations $\mathrm{S}$ and $\mathrm{K}$ in a somewhat different manner as seen in (b). The $\mathrm{X}$-ray images are mainly of $(\overline{1} \overline{1} 3)$ reflection partially overlapped by (002) reflection. The directions of striations $\mathrm{K}$ and $\mathrm{S}$, comparing with the optical micrograph (a), are found to be parallel to the deformation and primary slip bands, respectively.

Both striations $\mathrm{K}$ and $\mathrm{S}$ change with variation of the glancing angle. To find out about the minute shift of striations $\mathrm{S}$, a series of X-ray micrographs were taken at successive glancing angles using finegrained photographic plates. The fourth pattern (4) in Fig. 2 shows a further enlargement of the black-framed portion in Fig. 1 (b), on the left and right sides of which are arranged those taken in sequence at intervals of $0.05^{\circ}$ of the glancing angle in order to

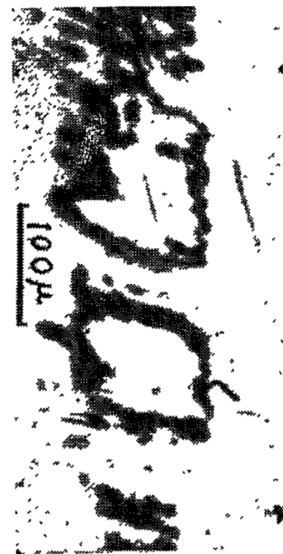

(1) $\alpha=5.10^{\circ}$

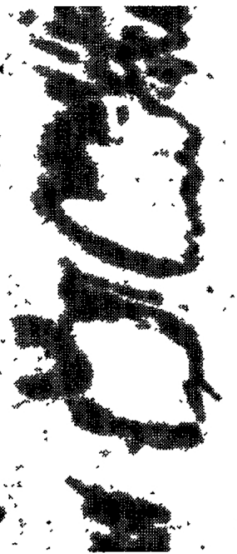

(2) $5.05^{\circ}$

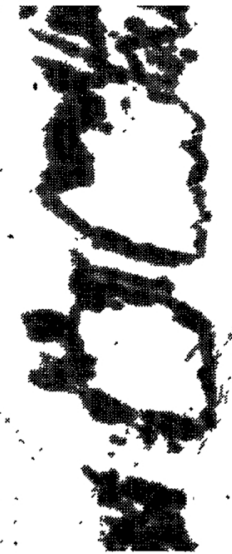

(3) $5.00^{\circ}$

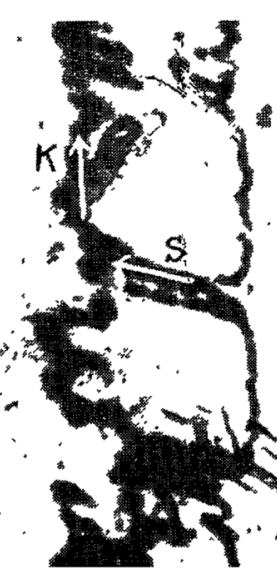

(4) $4.95^{\circ}$

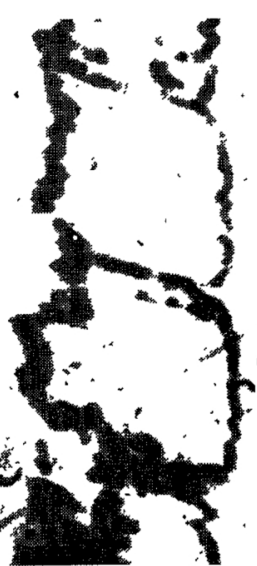

(5) $4.90^{\circ}$

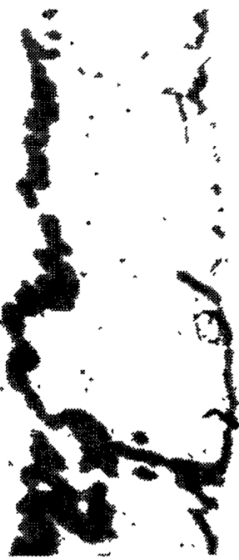

(6) $4.85^{\circ} \times 125$

Fig. 2 Change of Striations $S$ with the glancing angle in the case of parallel-setting ( 113 reflection). Specimen $148 \mathrm{~B}(\varepsilon=11 ; 0)$ 
take a view of the change of striations S. Small parallelograms seen in these micrographs consist of two couples of the neighbouring striations $\mathrm{K}$ and $\mathrm{S}$ which cut each other. One set on the opposite sides of the parallelogram due to striations $S$ (nearly borizontal on the micrographs) shifts laterally (vertically on the micrographs) with variations of the glancing angle, the shifting direction being opposite for the neighbouring atriations. Further, both end portions of striations $\mathrm{S}$ connected with striations $\mathrm{K}$ turn to the inside, each parallelogram being rounded at its corners.

Now one must consider the reason for these changes in behavior of striations S. Regarding the shift of striations $S$, it is noticed in the present case that there is an essential difference from that of striations $\mathrm{K}$. With variation of the glancing angle, each of the neighbouring striations $S$ shifts to the opposite direction as in the case of striations $K$, while the shifting process is discontinuous and at a certain glancing angle another striation $S$ appears between or near a set of striations $S$ simultaneously. These facts can be more apparently recognized in Fig. 3,* which was taken from the same portion as in Fig. 2 using the vertical line source of X-rays.

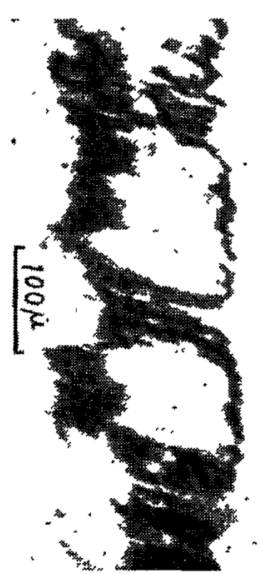

(1) $\alpha=5.35^{2}$

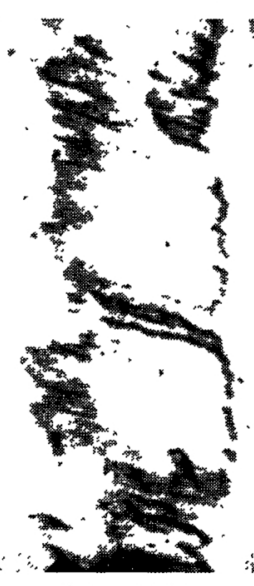

(2) $5.30^{3}$

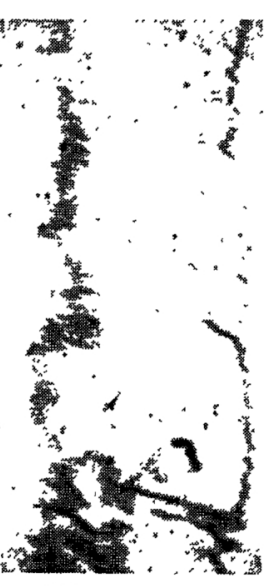

(3) $5.25^{\circ} \times 125$
Fig. 3 Resolution of striations $S$ in the case of perpendicularsetting, the same portion as in Fig. 2.

These facts cannot be interpreted in terms of the lattice bending with an rotation axis parallel to $\mathrm{S}$, as in the case of $K$, but the following two causes seem to be active;

(1) local lattice curvature at the slip band,

(2) twisting on the slip plane.

Concerning the occurrence of the local lattice curvature, a number of authors(4)(5)(6) have reported some evidences of it. Since the rasults of all these

* The values of glancing angle $\alpha$ noted in Figs. 2 and 3 are readings of the graduator fitted to the screw of the camera. For the value of $\alpha$, only the relative angular difference is reliable, since the irradiated portion under examination does not always satisfy the Bragg condition at the glancing angle of the same reading in both parallel and perpendicular settings.

(4) G. I. Taylor: Trans. Faraday Soc , 24 (1928), 121.

(5) H. Mark, M. Polanyi and E. Schmidt: Z. Physik, 12(1923), 58.

(6) K. Yamaguchi : Sci. Papers Inst. Phys. Chem. Res.,(Tokyo), $11(1929), 223$. investigations, however, were due to the fine structures of Bragg or Laue spots, the locality characters were deduced only by a speculation. Taylor and Yamaguchi independently concluded from their experiments that the axis of local lattice curvature lay on the slip plane and is perpendicular to the slip direction, while Polanyi et al. reached another conclusion that it is perpendicular to the tension axis and to the slip direction. On the formation mechanism of the local lattice curvature, Burgers proposed(7) a model, whose idea is adopted in Fig. 4. In the present

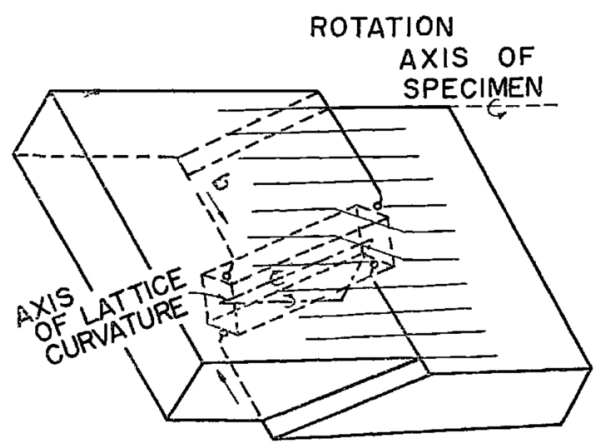

Fig. 4 Local curvature with the component along the rotation axis of specimen.

case, such an axis of lattice curvature according to their idea is not perpendicular to the rotation axis of the specimen, but inclined by $54^{\circ}$ or $62^{\circ}$, respectively, and the lattice curvature will be found to be appreciable on the X-ray image with variation of the glancing angle in view of either idea. In this way, if there exists portions of such local lattice curvature along the slip bands, the reflection image will appear along the slip bands, because of the strong reflection due to reduction of the extinction effect on these portions. The direction of striations $S$ seen in the micrographs supports the above interpretations. It is not now, however, determinable which is right TaylorYamaguchi's or Polanyi's, because the value of $\eta$ is small in the specimen used. The discontinuous lateral shifts of striations $S$ as stated above can also be approved by this locality character. In addition, striations $S$ are not always continuous but spotty in the longitudinal direction especially in the case of the "parallel" setting as seen in Fig. 2. This fact is due to the local lattice curvature at the slip bands, and the actual size of this bent portion in the specimen may be far less than the image as seen in Figs. 2 and 3.

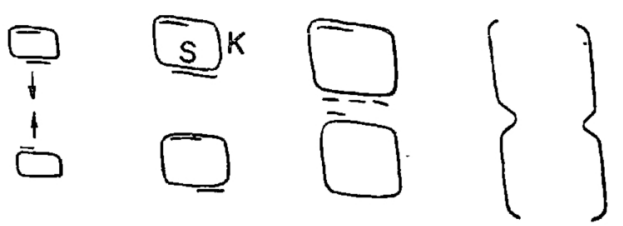

Fig. 5 Shif ting of striations $\mathrm{S}$ (schematically shown).

Thus the changing behavior of striations $\mathrm{S}$ can almost be explained by cause (1). And yet this behavior of striations $\mathrm{S}$ is accompanied by a gradual lateral long-range shift. Namely, as sketched in Fig. 5, when

(7) J. M. Burgers : Proc. Phys. Soc. (London), 52 (1940), 23. 
the space between opposite striations $\mathrm{S}$ in a parallelogramic image widens with variation of the glancing angle, two adjacent sides $\mathrm{S}$ of the neighbouring parallelograms approach each other and subsequently unite into a single line and at last disappear. This fact suggests the existence of a twisting on the slip plane. It seems that between the striations $S$ shifting upward and downward, there is a difference in the sense of the twisting moment and a cancellation of the twisting may occur when they are approached. The distance between the two neighbouring twisting regions is $10 \sim 100 \mu$, and the angle of twisting is estimated to be approximately $10^{\prime} \sim 20^{\prime}$ from the difference between angles at which striations $S$ appear and disappear.

A similar evidence for such twisting was also obtained experimentally by N.Kato(8) in a plastically deformed KCl crystal. He observed the twisting on (001) plane from the shift in Laue spot (200) obtained by Lambot's technique.(9) According to his result, the angle of twisting is $3.7^{\prime} \sim 16^{\prime}$ and the distance between the twisted regions which would cancel out when they are approached is approximately $2 \mathrm{~mm}$. Kitajima(10) also obtained another evidence for such twisting by means of X-ray microbeam technique that there appeared the asterism in Laue spots from near the deformation band in an elongated $\mathrm{Al}$ crystal. In the last report ${ }^{(3)}$, the authors noticed another evidence of twisting. From the data obtained there, the angle of twisting was estimated to be approximately $30^{\prime}$. In that case, however, the positive and negative twistings were not perfectly canceled out. Further more it is generally found that the striations $K$ are not straight but seen to be ragged with some relations to striations $S$, and this fact can be interpreted to be due to the appearance of minute amounts of twistng. The round corner of

\section{(b) Clustered slip}

(i) Directions of striations in the $\mathrm{X}$-ray micrograph

In the previous report ${ }^{(\varepsilon)}$, rough estimation was made for the correspondence between the lamellar patterns in the X-ray image obtained and the strain markings in the optical micrograph. Since the X-ray image taken by the present technique is inevitably a little distorted (11) in responce to the reflecting net plane chosen, it is necessary to make correction for that distortion in order to get the precise correspondence to the optical micrograph.

In the present case, it will be approximately correct to assume that each of the incident and reflected beams is parallel itself (cf. p. 134 in the previous report).(3) So one can calculate the direction of the image line corresponding to the line on the specimen surface, using the direction cosines of the reflected beams (cf. Appendix of the previous report). (3) The measurement of these cosines was made by the outward tracing method*. That is, they were estimated from the amount of shift of the X-ray images when the photographic plate was displaced apart from the specimen, being kept parallel to the incident beam. The values thus calculated of the directions of the images were compared with the experimentally measured ones of the directions of striation patterns seen in the X-ray image. They are given in Table 2, from which it is seen that the directions of striations $\mathbf{K}$ and $S^{\prime}$ are parallel to the kink bands and the bands of secondary slips respectively within the experimental error, while, striations $S_{1}$ and $S$. are inclined to the primary and secondary slip bands respectively by several degrees, which is larger than the experimental error. The direction of striations $S_{2}$ seen in the secondary slip region was unable to be accurately

Table 2 Distortion of the strain figure.

\begin{tabular}{|c|c|c|c|c|c|c|c|c|}
\hline & \multirow{3}{*}{$\begin{array}{c}\text { Optically } \\
\text { Measured on } \\
\text { Specimen Surface } \\
(\chi)\end{array}$} & \multicolumn{7}{|c|}{ X-ray Reflection } \\
\hline & & \multirow{2}{*}{$\begin{array}{l}\text { Kind of } \\
\text { Striations }\end{array}$} & \multicolumn{2}{|c|}{$(1 \overline{1} 1)$} & \multicolumn{2}{|c|}{$(1 \overline{3} 1)$} & \multicolumn{2}{|c|}{$(3 \overline{1} 1)$} \\
\hline & & & $\begin{array}{l}\text { Expected } \\
\text { from } \chi\end{array}$ & Measured & $\begin{array}{l}\text { Expected } \\
\text { from } \chi\end{array}$ & Measured & $\begin{array}{l}\text { Expected } \\
\text { from } \chi\end{array}$ & Measured \\
\hline Primary Kink Band & $-53.6^{\circ} \sim-55.0^{\circ}$ & $\mathbf{K}$ & \begin{tabular}{|}
$-55.0^{\circ} \sim$ \\
$-56.3^{\circ}$
\end{tabular} & $-55.0^{\circ}$ & $\left|-\frac{53.1^{\circ} \sim}{-54.6^{\circ}}\right|$ & $-54.5^{\circ}$ & \begin{tabular}{|}
$-55.2^{\circ} \sim$ \\
$-56.6^{\circ}$
\end{tabular} & $-56.8^{\circ}$ \\
\hline Primary Slip Trace & $38.7^{\circ}$ & $\mathrm{S}_{1}$ & $42.7^{\circ}$ & $47.8^{\circ}$ & $38.5^{\circ}$ & $46.0^{\circ}$ & $40.1^{\circ}$ & $45.0^{\circ}$ \\
\hline Secondary Slip Trace & $-28^{\circ}$ & $\mathrm{S}_{2}$ & $-30^{\circ}$ & $-24^{\circ}$ & $-28^{\circ}$ & $-18^{\circ} \sim-27^{\circ}$ & $-29.2^{\circ}$ & $-15^{\circ} \sim-18^{\circ}$ \\
\hline $\begin{array}{l}\text { Band of Secondary } \\
\text { Slips }\end{array}$ & $32.7^{\circ} \sim 35.5^{\circ}$ & $\mathrm{S}^{\prime}$ & $36.3^{\circ} \sim 39.3^{\circ}$ & $37.3^{\circ} \sim 39.6^{\circ}$ & $32.6^{\circ} \sim 35.3^{\circ}$ & $31.4^{\circ} \sim 32.9^{\circ}$ & $34.1^{\circ} \sim 36.8^{\circ}$ & $34.7^{\circ} \sim 35.2^{\circ}$ \\
\hline
\end{tabular}

the parallelogramic image seems to be due to the resultant effect of the lattice bending in deformation bands and the twisting stated above.

(8) N. Kato: Acta Met., 5 (1957), 237.

(9) H. Lambot, L. Vassamillet and J. Dejace: Acta Met., 1 (1953), 711.

(10) S. Kitajima : Metal Physics, (Japan), 3 (1957), 29.
(11) M. Yamamoto, M. Doi and Z. Nishiyama: J. Appl. Phys. Japan, 20 (1951), 257.

* By this method it is also possible to check whether the image obtained is due to the reflection of the chosen net plane or not. The specimen was mounted in the camera with intention to get the reflected beams to be in the equatorial plane (XY-plane) as near as possible, but af ter this procedure, it was found that the reflected beams were of ten inclined by several degrees of arc to the equatorial plane. 
measured. It was difficult especially in the case of $(1 \overline{1} 1)$ and $(1 \overline{3} 1)$ reflection so that even the determination of its direction could hardly be performed. For striations $S_{1}$, bowever, the above stated inclination must be accurate, since striations $S_{1}$ are straight and have a common direction. The description concerning striatons $S_{1}$ will be given again in the later section.

\section{(ii) Characters of striations $\mathbf{S}^{\prime}$}

In order to clarify the nature of striations $\mathrm{S}^{\prime}$, the $\mathrm{X}$-ray micrographs were taken of $(1 \overline{3} 1)$ reflection using Specimen $\mathrm{A}_{0}-\mathrm{IV}-4(\varepsilon=5 \%)$ with variation of the glancing angle. They are partly shown in Fig. $6(\mathrm{~b}) \sim$ as for striations $S_{b}$ as denoted formerly(2), each of them originates from a secondary slip region which is rather wider than the $S^{\prime}$ region, and it corresponds to a bundle of slip clusters whose distribution appears to be bandy in the case of lower magnification. The striation $\mathrm{S}$ also appears in the secondary slip region. Referring to the optical micrograph, it is found to correspond to a small primary slip region occurring within the secondary slip region.

Now let us look into striations $\mathrm{S}$ more closely. Detailed inspection of Fig. 6 enables us to find that $\mathbf{S}^{\prime}$ changes in its reflection patterns with variation of the glancing angle. These changing aspects

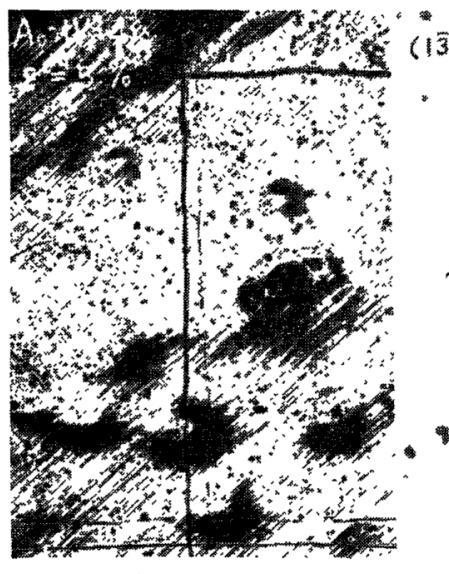

(a) Optical

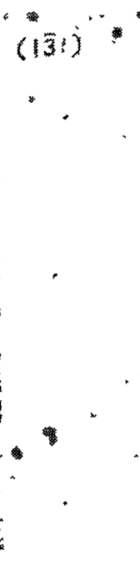

(b) $\alpha=6.6^{\text {? }}$

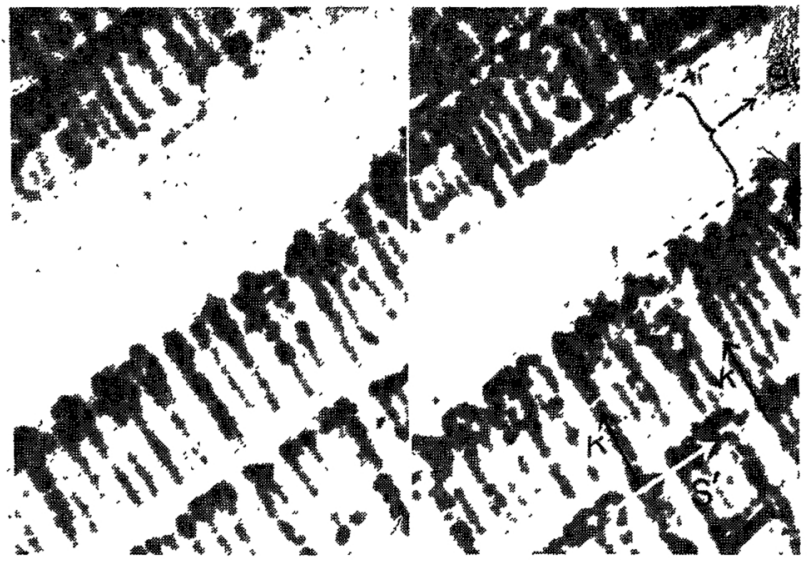

(c) $6.8^{\circ}$ (d) $6.9^{\circ}$

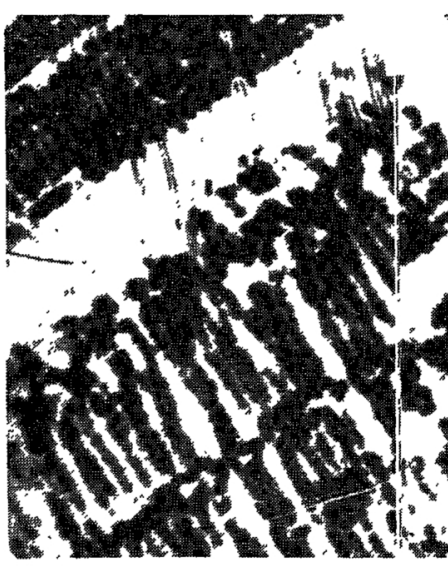

(e) $7.0^{\circ}$

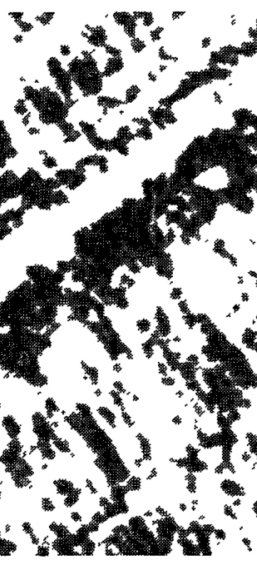

(f) $7.1^{\circ}$
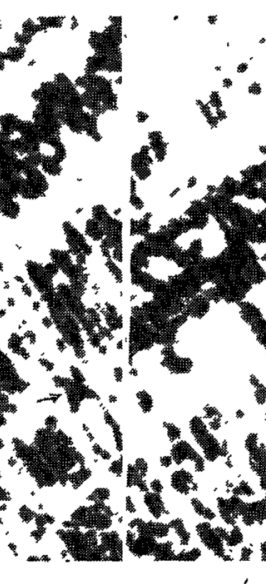

(g) $7.2^{\circ}$
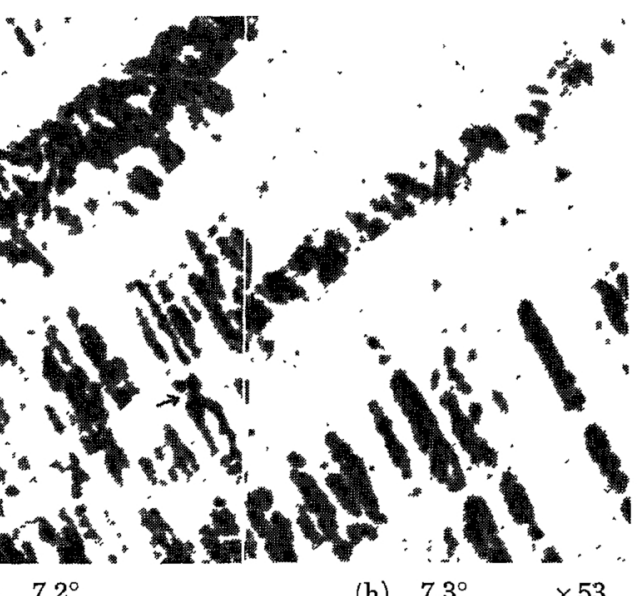

Fig. 6 Change of striations $S^{\prime}$ with the glancing angle, (Specimen $\left.A_{0}-\mathbb{V}-4, \varepsilon=5 o^{\prime}\right)$. (b $\sim \mathrm{h}:(1 \overline{3} 1)$ reflection)

(h), which are arranged in sequence at intervals of $0.1^{\circ}$ or $0.2^{\circ}$ of the glancing angle. Comparing these X-ray micrographs with the optical one (a), paying attention to the inside of a striation of $S$ (shown in (d)), it is generally found that in (a) the primary slips appear sparsely at the portion corresponding to the striation $\mathrm{S}^{\prime}$, although in some regions the secondary slips are slightly visible. From this comparison and the directional behavior seen in Table 2 , it is concluded that a striation of $S^{\prime}$ corresponds to a small region where the principal slips are few but rather secondary slips have taken place within the primary slip region*. Also

* In the previous paper ${ }^{(2)}$, it was only roughly stated that the lower half of the X-ray micrograph (c. f. Photo. 6 in the paper) was the primary slip region having striations $\mathrm{S}^{\prime}$, the nature of which remained in question. are schematically shown in Fig. 7, in wbich there is a secondary slip region sandwiched between two primary slip regions. The change of striations $\mathrm{K}$ in the primary region is as described formerly, ${ }^{(3)}$ that is, when the glancing angle is small (figure A) the reflection of X-rays is strong at the portion of kink bands but as the glancing angle increases (to figure E) the reflecting portion shifts to the simply slipped region between the neighbouring kink bands. Regarding the angular range in which the image appears, it is wider for the kink band portions than for the intermediate ones. By further inspection of Fig. 6 (f), (g), which were taken at glancing angles capable of the reflection from large secondary regions, it is found that the reflection image of $S^{\prime}$ appears to connect the head portions of two opposite K's as 
indicated by arrows. Considering this with the above facts, it is clear that $S$ is due to the reflection from the secondary slip region. Therefore by analysing $S^{\prime}$ it will be possible to get a key to find the entity of the secondary slip.

In order to interprete the changing aspects of $\mathrm{S}^{\prime}$, a model as shown in Fig. 8 is assumed. In this figure,
On the secondary slip region, studies have been made by several authors(12)(13). Calnan presented an evidence that Laue spots from the portion in the secondary region (Striae) scarcely showed the asteriem, while those from the border portion of the secondary region appeared to be fragmented. The axis of lattice rotation for the fragmentation was considered to be

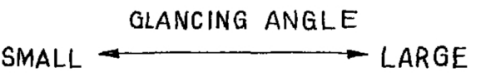

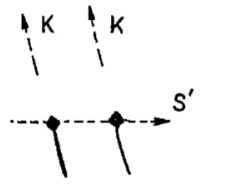

A

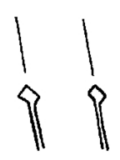

B

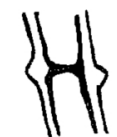

C

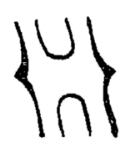

D
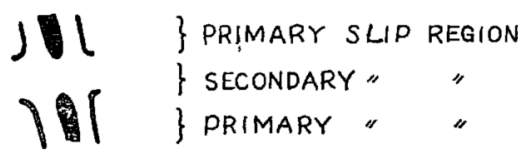

E

Fig. 7 Change of striations $\mathrm{S}^{\prime}$ with the glancing angle. (Schematically shown)

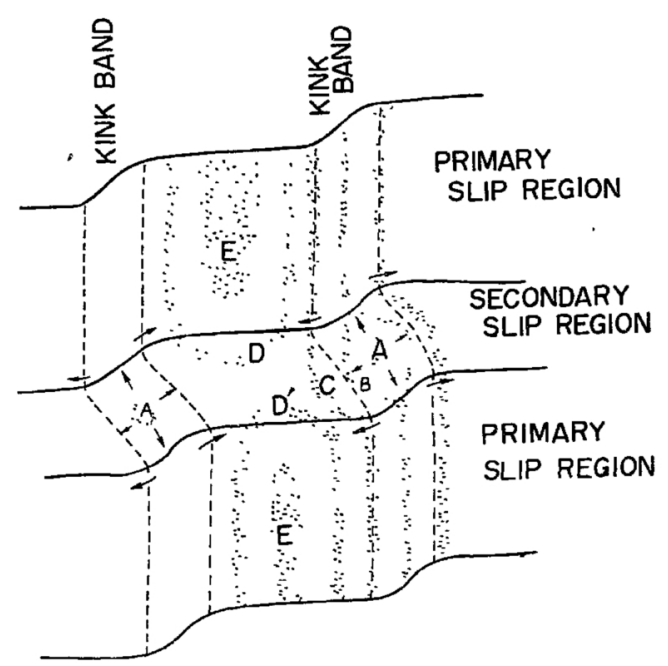

Fig. 8 Deformation model of the clustered slip: Dotied areas show the regions from which reflections occur in the case of $\mathrm{A}-\mathrm{E}$ in Fig. 7 in a sequence. Arrows show the direction of shift of the reflection with the glancing angle.

there is a secondary slip region sandwiched by two primary slip regions, between which the misorientation of lattice exists.* At the boundaries between them, local constraints are expected to occur. Hence the reflection will be strong near the boundaries as observed. If the lattice rotation due to these constraints is opposite for both sides of the boundary, the correspondence between Figs. 7 and 8 is good; with the sequence $(A, B, C, \cdots)$ of the variation of glancing angle in Fig. 7, dotted areas in Fig. 8 reflect the X-ray beams in turn. Arrow marks in Fig. 8 indicate the direction of shifting of reflecting portions. In this model, the rotation axis of lattice near the boundaries is not required to be the same as that of the kink bands in the primary region but it is enough if the rotation has a component parallel to the plane of paper.

* This was apparently observed also in the $\mathrm{X}$-ray reflection from another net plane (3ī1) of the same specimen. in the plane of the conjugate slip and to be perpendicular to the direction of the slip. These results are in agreement with the model stated above. Further, he optically observed the rumpled structure in the secondary region, and he suggested that this rumpled structure corresponded to deformation bands belonging to the secondary and tertiary slip systems. In the present experiment, however, in the secondary region there are found no kink bands belonging to the conjugate slip system but only the conjugate slips, by $\mathrm{X}$-ray micrographs as well as by the optical. The strain due to deformation is rather concentrated near the boundary between the primary and secondary slip regions, probably caused by the constraints due to the lattice deformation of both regions. Since the strain figure in the secondary region is quite complex and irregular, the lattice strain will be of the short range but large in magnitude, and therefore the complete analysis of the strain in the secondary region will be difficult. Nevertheless, the primary slip region, compared with the secondary one, is considered to have a larger bending strain of long range (of the order of the width of a kink band), since the angular range in which the image appears is wider for the former than for the latter.

\section{(iii) Characters of striations $\mathbf{S}_{1}$}

Figs. $9(\mathrm{~b}) \sim(\mathrm{f})$ are $\mathrm{X}$-ray micrographs of $(3 \overrightarrow{\mathrm{l}} \mathrm{l})$ reflection from Specimen $A_{0}-\mathrm{IV}-4$, which are arranged in sequence at intervals of $0.1^{\circ}$ of the glancing angle, (a) being the optical micrograph of the same portion. As stated formerly, striations $S_{1}$ are inclined by an angle of the order of $5^{\circ}$ to the primary slip trace, which is larger than the experimental error. By the optical means, any strain markings corresponding to these are not observed. Therefore the nature of striations $S_{1}$ seems to be different from that of striations $S$. Striations $S_{1}$ hardly change with variation of the glancing angle and the angular range in which $S_{1}$ appears is so narrow that the parallelism of incident

(12) R. W. K. Honeycomb : J. Inst. Metals, 80 (1951), 45.

(13) F. A. Calnan : Acta Cryst., 5 (1952), 557. 


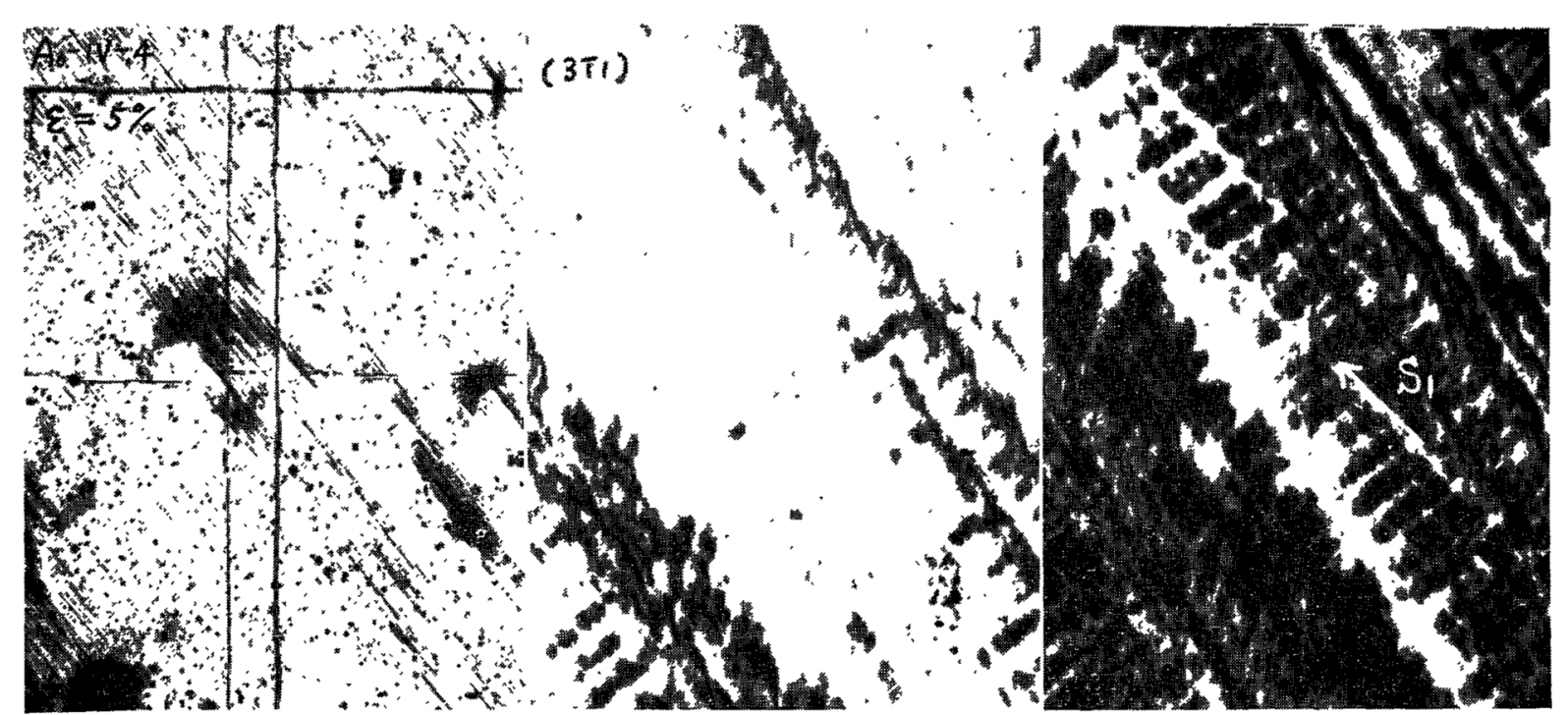

(a) Optical

(b) $\alpha=19.5^{\circ}$

(c) $19.6^{\circ}$

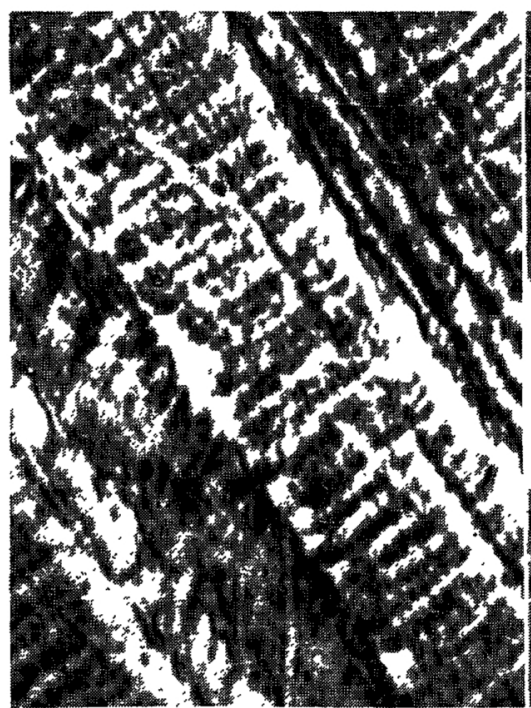

(d) $19.7^{\circ}$

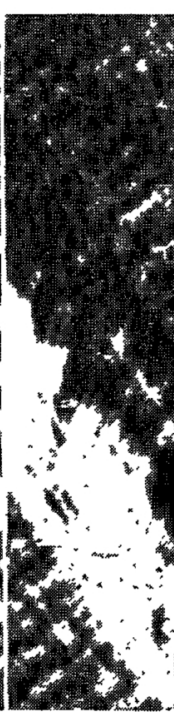

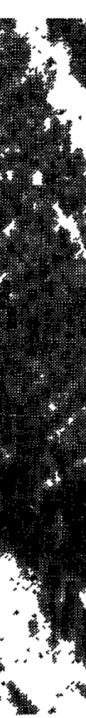

(e) $198^{\circ}$

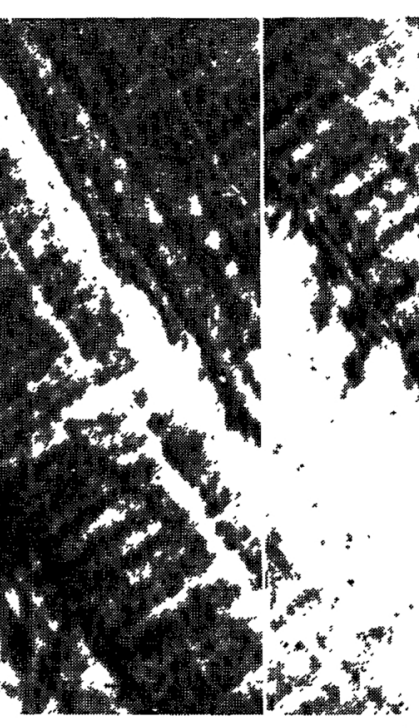

(f) 19.9

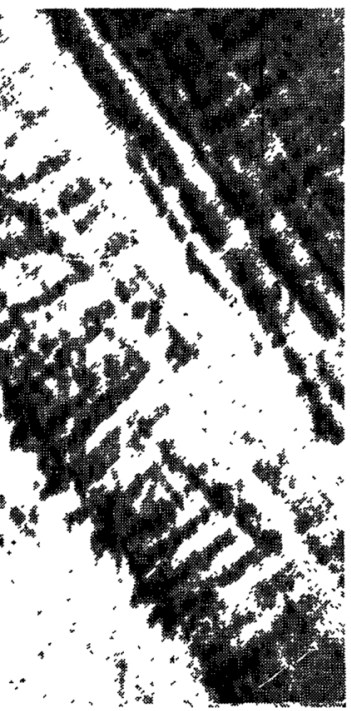

$\times 64$

Fig. 9 Change of striations $S_{1}$ with the glancing angle, (Specimen $\left.A_{0}-\mathbb{V}-4, \varepsilon=5 \%\right)$. (b $\sim f:(3\lceil 1)$ ref lection)

X-ray beams needs to be more strict in order to detect the fine variation.

As seen in the $\mathrm{X}$-ray micrographs given above, striations $K$ cut through striations $S_{1}$ and $S^{\prime}$, only being weakened in intensity at the intersections, while $\mathrm{S}_{1}$ does not cut through $\mathrm{S}^{\prime}$. These behaviors of the striations will be noticeable on considering the course of plastic deformation.

\section{Conclusion}

By the Berg-Barrett method, X-ray diffraction micrographs were taken of slip bands and of the clustered slips in elongated aluminium single crystals. On the slip bands, the following have been found:

(1) Striations S running parallel to the slip bands shift laterally with change of the glancing angle, the direction being opposite for the neighbouring striations. This shift can be interpreted as the existence of twists whose axes are perpendicular to slip planes and whose directions are opposite for the neighbouring slip bands. In addition it has been found that

(2) Striations $\mathrm{S}$ have locality characters: The spotty reflection and the discontinuous lateral shift. These characters show the existence of the local lattice curvature at the slip bands.

The X-ray micrographs taken from the clustered slip region showed four kinds of striation $\left(S_{1}, S, S^{\prime}\right.$, $\mathrm{S}_{\mathrm{b}}$ ) related to the active slips. Analysing these striations the following have been observed:

(1) Striations $S^{\prime}$ are found in both the primary and the secondary slip regions, the former corresponding to the small region of secondary slip taking place in the primary slip region, and the latter does vice versa.

(2) In the secondary slip region there are no kink bands belonging to the secondary slip system but the strain due to deformation is concentrated near the boundary between the primary and the secondary slip regions, probably caused by the constraints due to the lattice deformation in both regions.

(3) Striations $S_{1}$ are straight and, in the present case, inclined by several degrees to the primary slip trace, which is larger than the experimental error.

(4) The primary slip region, compared with the secondary one, has a larger bending strain of long range (of the order of the width of a kink band). 